\title{
Análise da Oscilação da Poça de Solda em P-GMAW por Meio de Processamento de Imagens Obtidas por Perfilografia
}

\author{
(Analysis of weld pool oscillation in P-GMAW by Means of Shadowgraphy Image Processing)
}

\author{
Esdras Godinho Ramos ${ }^{1}$, Guilherme Caribé de Carvalho ${ }^{1}$, Sadek Crisóstomo Absi Alfaro ${ }^{1}$ \\ ${ }^{1}$ Universidade de Brasília, Departamento de Engenharia Mecânica, Brasília,DF, Brasil, esdrasgr@gmail.com
}

\begin{abstract}
Resumo
Este artigo apresenta uma metodologia de estudo da oscilação da poça de solda que permite obter as frequências de oscilação da poça tanto por sinais de tensão quanto por análise das imagens. O intenso brilho do arco é reduzido utilizando-se a técnica de perfilografia, e as imagens são obtidas a 1000 fotos por segundo por uma câmera CMOS de alta velocidade. A geometria da poça é visualizada em perfil e são obtidos espectros de frequência por transformada rápida de Fourier por meio de processamento das imagens em MATLAB. O processo de soldagem experimentado foi o GMAW pulsado com e sem a chamada "corrente de destacamento". Os resultados mostram que é possível medir a oscilação da poça por esta metodologia e até mesmo pode ser mais confiável a quantificação da oscilação pelas imagens do que pela a análise do sinal de tensão.
\end{abstract}

Palavras-chave: GMAW Pulsado; Oscilação da Poça de Fusão; Filmagem em Alta Velocidade; Filtros Espaciais.

\begin{abstract}
This paper presents a methodology for studying the weld pool oscillation by means of measuring its oscillation frequencies using both the welding voltage signal and the analysis of weld pool profile images. The intense arc brightness is reduced by using the technique of shadowgraphy and images are obtained at 1000 frames per second by a high speed CMOS camera. The geometry of the pool is viewed in profile and frequency spectra are obtained by Fast Fourier Transform using MATLAB image processing. The methodology was applied to analyze the weld pool oscillatory behavior that results from a pulsed GMA welding process. Two different welding current pulse types were tested: a) the conventional trapezoidal pulse (with only one level of peak current) and b) the trapezoidal pulse with the so called "detaching current" (the welding current presents a modified trapezoidal shape, in which, at the end of the peak current time, the welding current is reduced to an intermediary level and kept in that during some time, after which it reduces further to the background current level). The results show that it is possible to measure the puddle oscillation using this methodology and, also, that the measurement by images present itself as more reliable than the analysis by the voltage signal.
\end{abstract}

Key-words: Pulsed GMAW; Weld Pool Oscillation; High Speed Filming; Spatial Filters.

\section{Introdução}

Com a evolução dos robôs industriais e sua crescente utilização nas indústrias em processos de soldagem robotizada, surgiu a idéia de desenvolver o processo de fabricação por deposição de metal em camadas sucessivas utilizando robôs soldadores. Um dos estudos com vistas ao seu desenvolvimento aplicado a protótipos do tipo casca utilizando soldagem a arco iniciou-se na Universidade de Cranfield, Inglaterra, em 1992. O projeto inicial era financiado por uma indústria aeroespacial local e visava à integração de técnicas de prototipagem rápida com o processo de soldagem robotizada a arco do tipo GMAW (Gas Metal Arc Welding) convencional, de forma a reduzir o tempo de desenvolvimento e produção do primeiro protótipo metálico de uma peça. Utilizando o processo tradicional de fundição,

(Recebido em 10/04/2012; Texto final em 16/08/2012). o tempo necessário para o desenvolvimento dos primeiros protótipos de peças semelhantes variava de seis a dezoito meses. Com o processo de prototipagem rápida esse tempo poderia ser reduzido a, no máximo, um mês, o que geraria uma economia considerável. Além disso, todo o trabalho necessário ao desenvolvimento dos moldes de fundição tornar-se-ia desnecessário. Atualmente, estudos sobre o uso de soldagem 3D e prototipagem rápida baseada em soldagem para fabricação de peças vêm sendo desenvolvidos na Universidade de Nottingham (Reino Unido), na Universidade de Wollongong (Austrália), na Universidade Metodista Sulista (EUA), em grupos de pesquisa na Coréia, Índia e Alemanha e na Universidade de Brasília (Brasil) financiada pelo Conselho Nacional de Desenvolvimento Científico e Tecnológico (CNPq).

Em estudos realizados sobre prototipagem rápida de sólidos tipo casca, com o processo GMAW [1, 2 e 3], observou-se que a deposição de metal utilizando o processo convencional, no modo de transferência de metal por curto circuito, resultava em um acabamento superficial das paredes do sólido depositado bastante irregular e rugoso. Com o processo pulsado (P-GMAW) observou-se que o acabamento era melhor, entretanto ainda com 
rugosidade excessiva. Uma hipótese sugerida pelos autores do presente artigo para explicar a rugosidade observada na superfície do sólido utiliza como base o regime de oscilação da poça líquida. O metal líquido da poça de fusão é excitado pelas variações instantâneas de tensão e de corrente de soldagem assim como pelo aporte de momentum proveniente do metal transferido do eletrodo consumível. Seu regime de oscilação é influenciado pelas forças inerciais dependentes do volume e do formato da poça, assim como pelas forças de tensão superficial e pelas características de resfriamento do metal líquido. Para verificar a hipótese, que existe forte correlação entre o regime de oscilação da poça e o acabamento superficial do sólido construído, faz-se necessário medir as frequências de oscilação da poça de fusão assim como correlacioná-las com a rugosidade superficial do sólido resultante. Neste artigo, descreve-se a metodologia desenvolvida para resolver a primeira parte do problema, isto é, medir as frequências de oscilação da poça de fusão, demonstrando sua eficácia em relação ao processo P-GMAW. O estudo completo do qual este artigo se origina pode ser lido na dissertação de mestrado de Ramos, E.G. [4]. O estabelecimento de correlações entre o acabamento superficial do sólido, construído pela deposição de metal de solda em camadas sucessivas, e as frequências de excitação provenientes do processo P-GMAW ainda serão objeto de pesquisa futura.

A principal referência para o desenvolvimento da metodologia apresentada neste artigo foi o trabalho de doutorado de Yudodibroto [5], em que se fez um estudo da oscilação utilizando o sinal de tensão e as imagens da poça. Neste, entretanto, não se mostrou um método computacional para medir a oscilação da poça pelas imagens. No mesmo trabalho [5], as imagens foram iluminadas utilizando lâmpadas halógenas que consomem muita energia. Também não se encontrou na literatura método de análise semelhante e utilizando apenas $1000 \mathrm{fps}$.

$\mathrm{O}$ texto que se segue apresenta uma breve introdução teórica sobre o processo P-GMAW, sobre os modos de oscilação da poça e sobre o método da perfilografia.

\subsection{Embasamento Teórico}

A qualidade da solda depositada pelo processo GMAW pulsado (P-GMAW) é muito dependente da característica do arco [6], e do comportamento da transferência de metal [7]. Mas o controle das características do arco e do comportamento da transferência de metal pela seleção apropriada dos parâmetros do pulso é bastante difícil devido à influência simultânea de um número relativamente grande de parâmetros durante a soldagem [8]. Isso envolve os parâmetros de pulso como corrente média $\left(\mathrm{I}_{\mathrm{m}}\right)$, corrente de pico $\left(\mathrm{I}_{\mathrm{p}}\right)$, corrente de base $\left(\mathrm{I}_{\mathrm{b}}\right)$, tempo de pico $\left(t_{p}\right)$, tempo de base $\left(t_{b}\right)$, frequência de pulso (f), assim como a tensão do arco (V). Entretanto, a dificuldade em se controlar os parâmetros de soldagem em P-GMAW pode ser amenizada ao se considerar a influência de um fator hipotético $\varphi$ (Equação 1) derivado do conceito de balanço de energia [9]:

$$
\begin{aligned}
\varphi=\left(I_{b} / I_{p} \cdot f \cdot t_{b}\right) & \\
& \text { onde } t_{b}=(1 / f)-t_{p} .
\end{aligned}
$$

Em Ghosh, P et. al. [10] demonstrou-se experimentalmente que as características do arco, com respeito à sua rigidez e às suas dimensões, (Figura1) podem ser significativamente controladas pela variação de, $\mathrm{I}_{\mathrm{m}}$ e tensão do arco seguindo relações empíricas. É importante destacar desse estudo que o comprimento do arco (L), o diâmetro da raiz do $\operatorname{arco}\left(D_{R}\right)$ e o diâmetro da base do arco $\left(\mathrm{D}_{\mathrm{p}}\right)$ aumentam significativamente com o aumento de $\varphi$ para qualquer valor de $\mathrm{I}_{\mathrm{m}}$. Enquanto que para um dado $\varphi$ e tensão de arco, o aumento de $I_{m}$ reduz o comprimento do $\operatorname{arco}(\mathrm{L})$ mas aumenta os diâmetros da raíz $\left(\mathrm{D}_{\mathrm{r}}\right)$ e da base $\left(\mathrm{D}_{\mathrm{p}}\right)$.

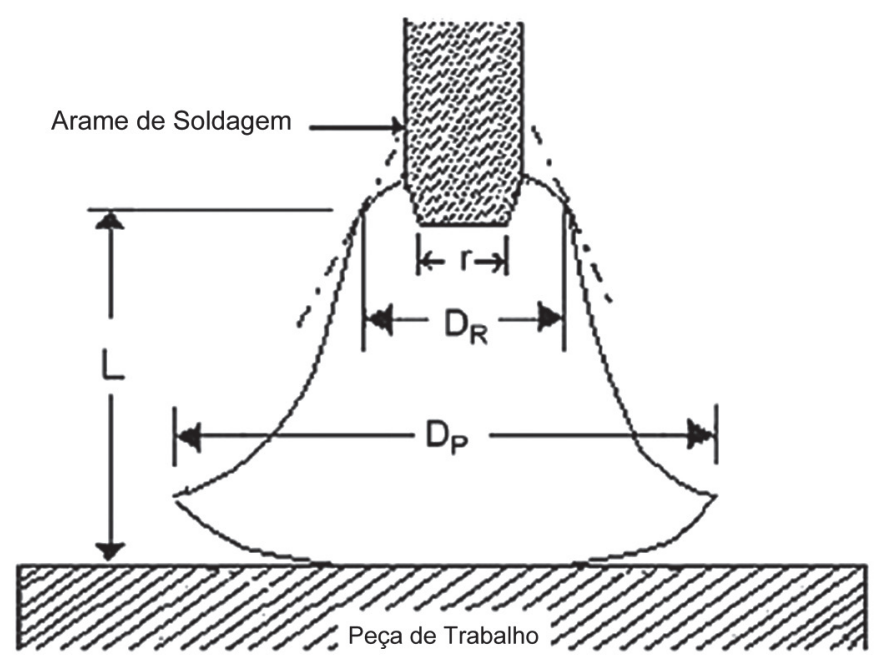

Figura 1. Diagrama esquemático mostrando as diferentes dimensões do arco (adaptado de [10]).

Nesse mesmo estudo [10], observou-se também que o arco é mais estável para valores de $\varphi$ menores do que para valores maiores. A estabilidade do arco é relacionada com a deflexão de sua parte mais brilhosa. Ou seja, para $\varphi$ maiores, maior é a deflexão do arco e menor é sua estabilidade.

A rigidez do arco é importante para evitar sua deflexão e está diretamente relacionada à sua pressão. A pressão do arco $\left(\mathrm{P}_{\mathrm{a}}\right)$ pode ser estimada com a ajuda de uma equação derivada da distribuição da pressão total de uma fronteira perturbada em uma interface líquido-sólido [11], por assumir o arco como um canal conduzindo um fluido cilíndrico de raio interno e externo igual ao raio de equilíbrio do metal fundido (R) e ao raio da raiz do $\operatorname{arco}\left(R_{a}\right)$, respectivamente [12]. A pressão do arco no processo P-GMAW pode ser expressa conforme mostra a Equação 2:

$$
P_{a}=\frac{\mu_{0} I_{P}{ }^{2}}{4 \pi^{2} R_{a}^{4}} \cdot\left(R_{a}^{2}-3 R^{2}+\frac{2 R^{3}}{R_{1}}\right)
$$

em que $R$ e $R_{1}$ são assumidos como sendo o raio da gota de diâmetro $\mathrm{D}$ e raio efetivo (r) da ponta do eletrodo respectivamente. Procedimentos empíricos levaram à conclusão que quanto maior $\varphi$ e $I_{\mathrm{m}}$, menor a pressão do arco (vide Figura 2). 

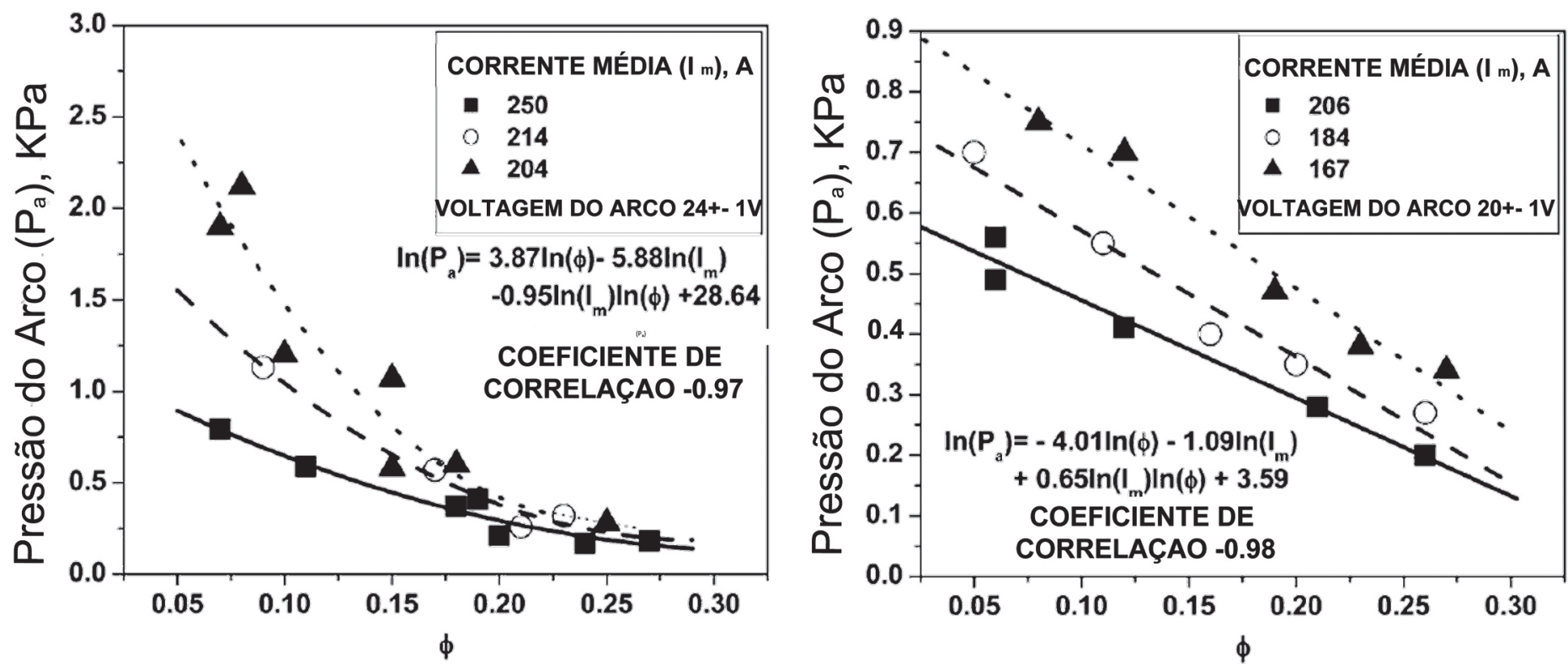

Figura 2. O efeito de $\varphi$ na pressão do $\operatorname{arco}(\mathrm{Pa})$ a diferentes Im, durante o tempo de pico nas tensões de 24V e 20V (adaptado de [9]).

\subsubsection{Modos de Oscilação}

No modo pulsado, um pico de corrente implica também em um pico de tensão e, como foi visto na Equação 2, a pressão do arco aumenta proporcionalmente ao quadrado da corrente de pulso e deforma a poça. Pesquisas sobre a oscilação da poça, como em [5], [13], [14] e [15], fundamentaram seus estudos com experimentos no processo GTAW, pelo fato deste possibilitar a visualização dos modos de oscilação com interferência apenas do arco e não do metal de adição. Essas investigações mostraram que a poça de solda pode oscilar em vários modos, alguns mais dominantes que outros. Também foi constatado experimentalmente que, no caso de penetração total, a poça oscila com frequências menores que em situações de penetração parcial. Xiao e den Ouden [13] propuseram que, com penetração parcial da poça de solda, apenas dois modos de oscilação são dominantes. Um deles possui uma oscilação simétrica com o centro da poça e o outro é assimétrico com a crista da onda oscilando em torno do centro da poça. Os mesmos autores [13] também propuseram que no caso de uma penetração total, a oscilação da poça de solda é dominada por um modo de oscilação assimétrico. Essas oscilações dominantes são mostradas na Figura 3.

\subsubsection{Perfilografia}

Um grande problema na visualização de um processo de soldagem é a alta intensidade luminosa emitida pelo arco, principalmente no processo GMAW por causa das altas correntes utilizadas. Quando não se pretende ver o arco e sim a deposição de metal, pode-se utilizar a técnica da perfilografia (shadowgraphy) [16]. Nesta técnica, uma fonte de luz como um laser ilumina a região que se deseja observar, de modo que a imagem de interesse seja a sombra dessa região. O feixe de luz é normalmente ampliado com o auxílio de um arranjo de lentes.
Do outro lado, uma câmera com filtro passa-banda captura a luz que está na região de comprimento de onda do laser e o que não estiver nessa região fica escuro, como a sombra do metal sendo depositado (Figura 4).



Figura 3. Modos de oscilação dominantes da poça de fusão (adaptado de [12]).

$\mathrm{O}$ arranjo da Figura 4 utiliza um expansor de feixe laser de tipo Galileu. Segundo Weichel [17] e Steen [18], o expansor utiliza uma lente divergente como entrada do feixe e uma convergente como saída, que de acordo com Hecht [19] produz idealmente uma frente de onda plana na saída do expansor, não tendo assim distorção nem ampliação nas geometrias dos elementos.

A frente de onda plana se consegue quando as frentes de onda esféricas de luz que entram na lente convergente partem do ponto focal objeto da mesma. Depois de atravessar a lente os raios ficam paralelos ao eixo óptico e as frentes de onda perpendiculares ao mesmo, como se vê na Figura 5. Nesse caso, os pontos focais das duas lentes se coincidem e diz-se que $o$ feixe de luz é colimado. 


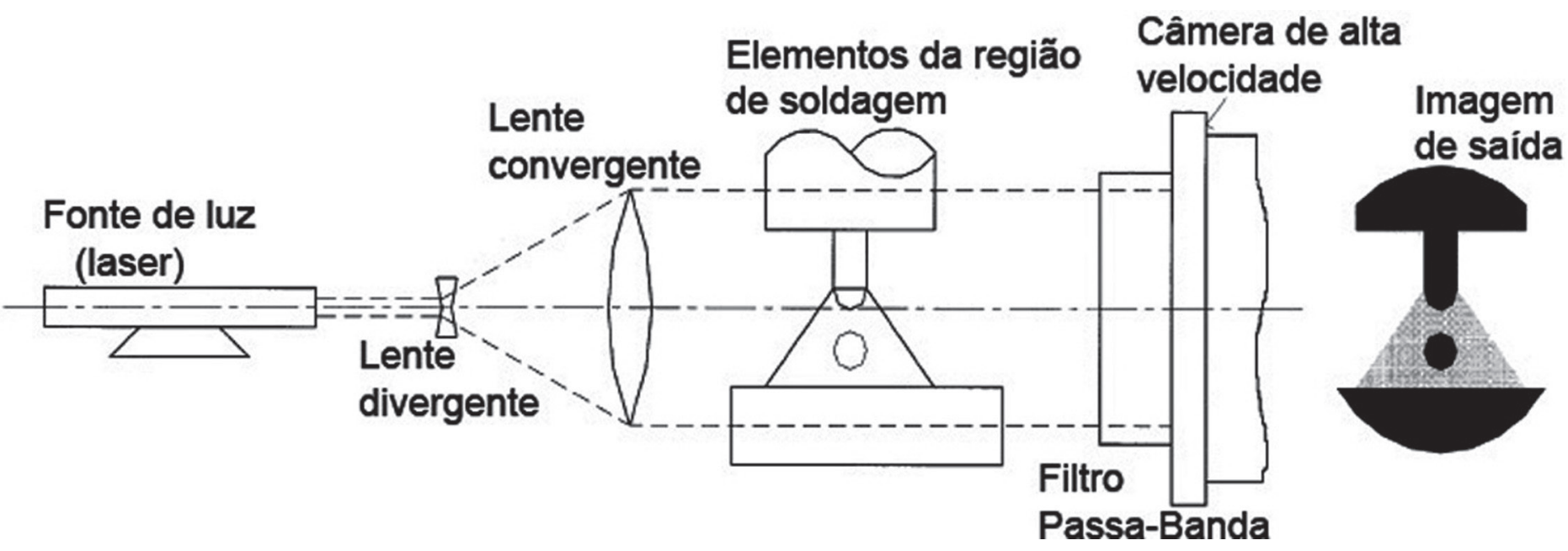

Figura 4. Princípio da perfilografia aplicado à soldagem [12].

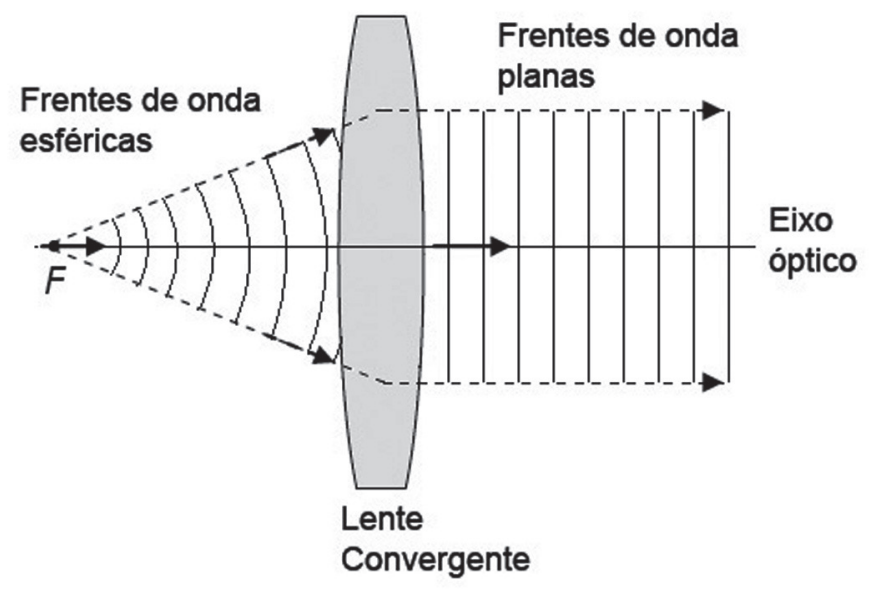

Figura 5. Formação de ondas planas a partir de ondas esféricas mediante o uso de uma lente convergente. A letra $F$ representa o ponto focal da lente. A luz incidente se propaga na direção das setas [20].

\subsection{Objetivos}

Nesta pesquisa, pretende-se desenvolver uma metodologia de medição das frequências de vibração da poça de fusão de modo a possibilitar em futuros trabalhos o estudo sobre os efeitos dessas frequências no acabamento superficial do sólido construído por Soldagem 3D. O que se espera observar nas próximas pesquisas é a relação entre o acabamento obtido pela deposição e as frequências de oscilação da poça. A hipótese a ser investigada nesse caso é se a regularidade das frequências de excitação pode ser responsabilizada pelo melhor acabamento superficial obtido no modo pulsado se comparado com o modo de transferência por curto-circuito. Além disso, a análise da oscilação da poça de solda permite inferir outros dados relativos à qualidade da solda a fim de incrementar a malha de realimentação, como a respeito de sua penetração.

\section{Aparato Experimental}

\subsection{Sistema Óptico}

A fonte de luz utilizada é um laser de $\mathrm{He}-\mathrm{Ne}$ com comprimento de onda de $632,8 \mathrm{~nm}, 15 \mathrm{~mW}$ de potência, que emite um feixe de luz com 1,2 mm de diâmetro. Para expandir o feixe utilizou-se um expansor de feixe de Galileu com uma lente divergente e uma convergente com distâncias focais de respectivamente $12,5 \mathrm{~mm}$ e $500 \mathrm{~mm}$. O diâmetro final do feixe colimado é de $48 \mathrm{~mm}$ (Figura 6).

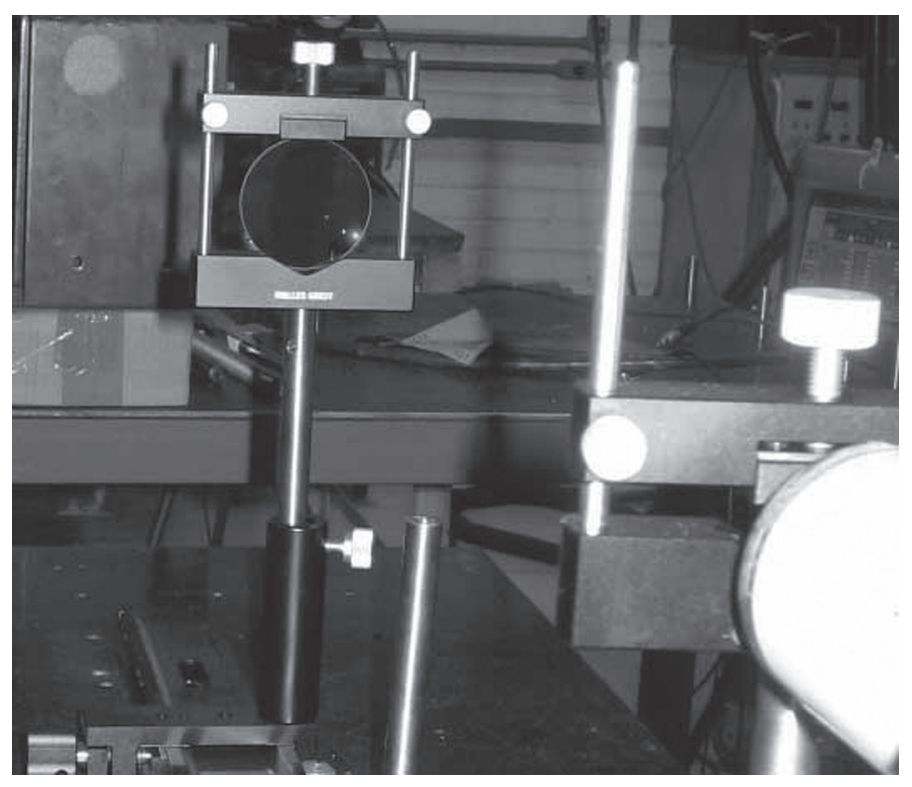

Figura 6. Feixe de laser colimado.

As imagens são capturadas através de uma câmera DALSA 1M150-SA de tecnologia CMOS a 1000 frames por segundo (fps). É uma câmera de alta velocidade e baixo custo em 
relação a outras utilizadas em monitoramento de processos de soldagem. A uma resolução máxima de 1 megapixel (1024x1024) ela permite capturar a uma taxa de até $150 \mathrm{fps}$. Seu pequeno tamanho e robustez a torna adequada para ambientes industriais. Ela também permite limitar a área de interesse que será fotografada, de forma que se reduza a resolução e aumente a taxa de aquisição, assim, para uma resolução de $256 \times 256$ pixels pode-se atingir uma taxa de captura teórica de $2200 \mathrm{fps}$. $\mathrm{Na}$ frente da lente da câmera é colocado um filtro óptico passa-faixa na região de comprimento de onda do laser.

\subsection{Elementos de Soldagem}

As soldas foram realizadas com a fonte de soldagem TransPuls Synergic 5000 da Fronius. O gás utilizado era composto de $96 \%$ de $\mathrm{Ar}$ e $4 \% \mathrm{CO}_{2}$. Como eletrodo consumível, utilizou-se o arame tubrod 410NiMo MC de $1.2 \mathrm{~mm}$ de diâmetro. Material de base de aço 1020. A soldagem era feita sobre uma mesa linear que se deslocava à velocidade constante e a tocha era posicionada com o auxílio de um braço robótico. Deve-se observar que o objetivo deste trabalho era somente desenvolver metodologia para medição das frequências de oscilação da poça de fusão, motivo pelo qual não se deu importância à seleção dos materiais. O arame Tubrod 410NiMo MC fora utilizado simplesmente por já estar instalado na máquina, assim como o gás de proteção.

\subsection{Elementos de Captura e Processamento de Dados}

Para fazer a sincronização das imagens obtidas com os sinais elétricos capturados nos experimentos, utilizou-se um

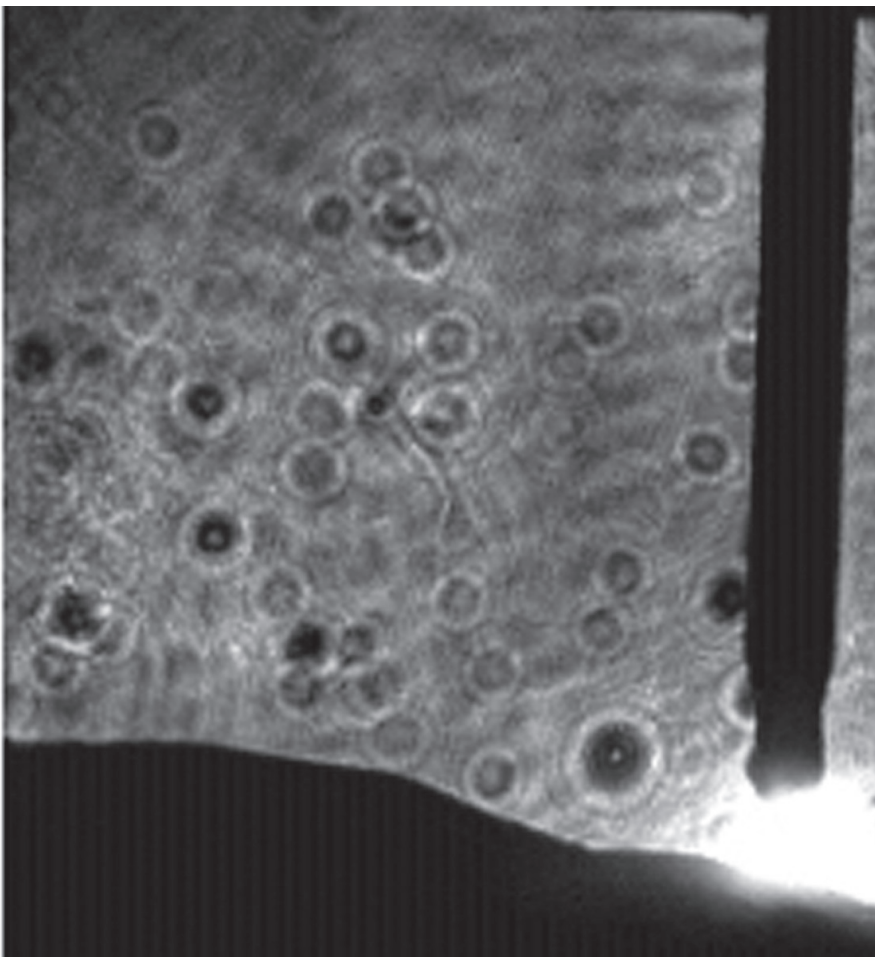

sinal fornecido pela câmera. Este sinal se apresenta como um pulso elétrico cada vez que uma foto é adquirida. A duração do pulso era de $50 \mu \mathrm{s}$. O valor do pulso varia ao redor de $3 \mathrm{~V}$. Os sinais elétricos capturados nos experimentos foram a corrente e a tensão de soldagem, o sinal que provém da câmera e, ainda, o sinal proveniente de um sensor de nível de luminosidade na faixa do ultravioleta. Esses sinais eram adquiridos por meio de uma placa de aquisição de dados a uma taxa de $20 \mathrm{kHz}$, e eram visualizados e armazenados por meio de uma interface desenvolvida em LabVIEW. As imagens adquiridas eram posteriormente processadas em um aplicativo desenvolvido em MatLab.

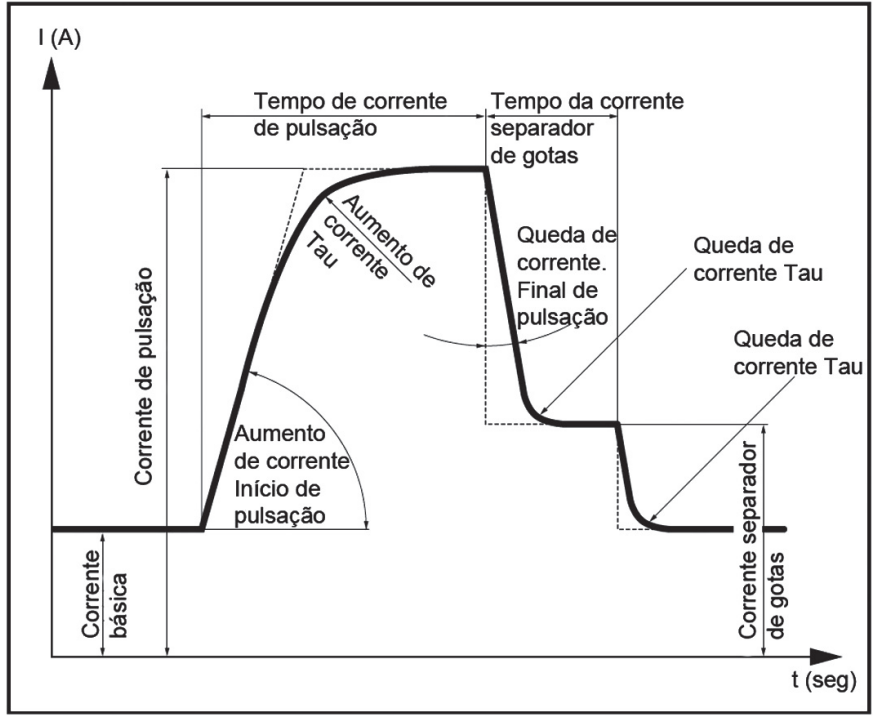

Figura 7. Parâmetros de pulsação da fonte de soldagem [21].

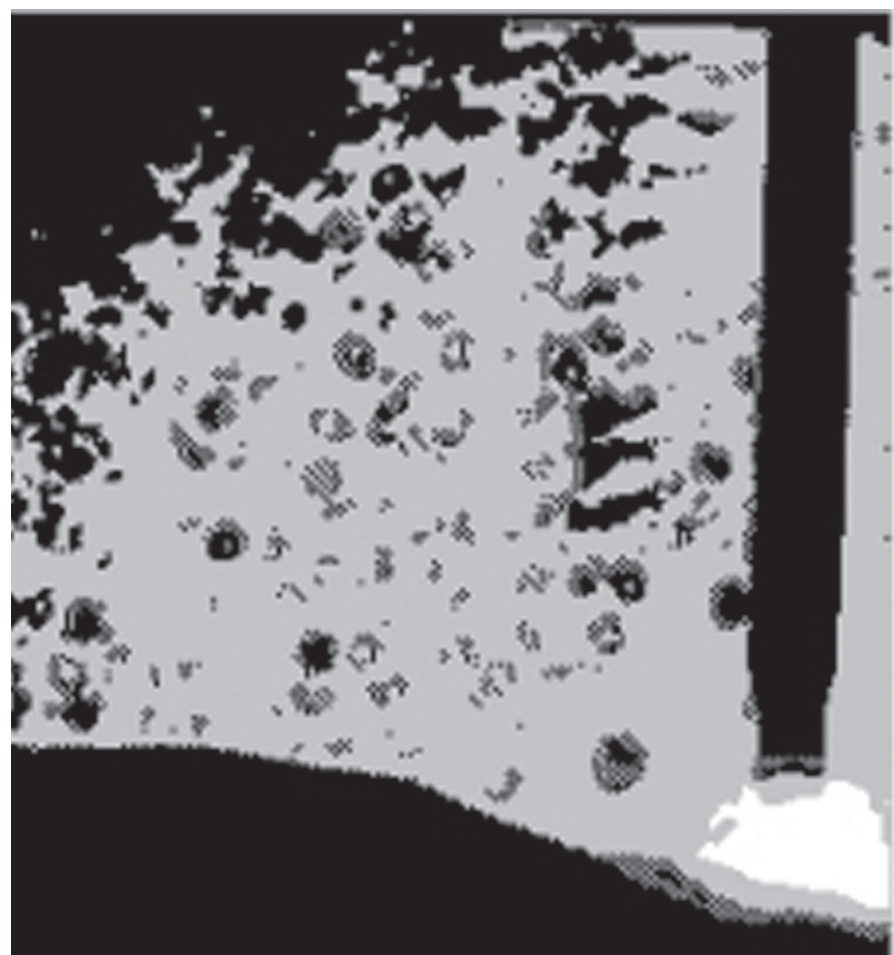

Figura 8. (a) Exemplo de Imagem Original (b) Exemplo de Imagem nivelada. 


\section{Metodologia}

O método de análise parte do princípio de que qualquer sinal periódico pode ser decomposto em somas de funções harmônicas senoidais e cossenoidais com frequências e amplitudes distintas. No modo de transferência GMAW pulsado, a fonte de soldagem gera pulsos de corrente na forma da Figura 7.

A corrente pode ser considerada como indutora das oscilações, já que induz o arco elétrico. Mas a tensão é fortemente influenciada pelo comprimento do arco, que é alterado com as oscilações da poça. Por isso, procura-se analisar principalmente o sinal de tensão.

Uma vez obtido o sinal de tensão, subtrai-se deste o valor médio correspondente e calcula-se o espectro de Fourier, em que as frequências mais significativas para a composição do sinal se apresentam com maiores amplitudes.

O próximo passo é verificar a oscilação da poça, por meio das imagens, e visualizar seu comportamento na formação do cordão de solda. Para isso, utilizou-se a técnica de perfilografia. A medição da oscilação a partir das imagens foi feita por meio da determinação da posição vertical do elemento da imagem correspondente à crista das ondas formadas. As imagens foram processadas de modo a se destacar o cordão em formação. Um filtro de mediana era usado na imagem original para eliminar pequenos ruídos, depois um filtro de derivada realçava as bordas a fim de estabelecer limites entre os elementos da imagem e, por último, realizava-se um nivelamento da imagem de modo a reduzir o número de níveis de cinza de 256 para somente 3 , de acordo com limiares pré-estabelecidos. A Figura 8 mostra exemplos referentes ao caso de imagem com 256 níveis de cinza e ao resultado após a realização do nivelamento.

Um outro filtro por derivadas comumente utilizado em processamento de imagens é o filtro de Sobel [22]. Esse filtro também pode ser aplicado para destacar o contorno do cordão de solda. Com a utilização deste filtro não foi preciso nivelar a imagem para obter a oscilação da poça.

Quando um ponto (pixel) é escolhido na imagem, no interior da região onde se encontra o metal líquido, o programa armazena sua coordenada horizontal e procura verticalmente (dentro do vetor formado pelos elementos de imagem com a mesma coordenada horizontal) o limite superior ou a margem referente ao limiar entre a superfície da poça e o gás de proteção. A coordenada vertical deste limite é então armazenada em um vetor. O mesmo procedimento é feito para as imagens subsequentes, de modo que o vetor de coordenadas verticais assim extraídas possa representar a característica oscilatória da superfície da projeção lateral da poça correspondente à coordenada horizontal previamente escolhida. A união desses pontos forma um gráfico oscilatório que corresponde à oscilação da crista da onda.

Há dois modos de se calcular a frequência de oscilação: um considera a oscilação de uma coordenada fixa no cordão e o outro, a coordenada variável de um ponto no cordão em movimento horizontal devido ao deslocamento da mesa. Para o segundo caso, o programa compensa o deslocamento linear da mesa. Para isso tem que conhecer quantas fotos são necessárias para que um ponto se desloque de um pixel no sentido horizontal. O tamanho do pixel é calculado com referência ao diâmetro do arame e, para saber quanto a mesa se desloca a cada foto, tem-se que conhecer sua velocidade linear, que corresponde à velocidade de soldagem, considerando o fato que a tocha é fixada no aparato experimental. Um algoritmo armazena como saída um vetor com as coordenadas do ponto móvel em cada uma das imagens analisadas. Por último calcula-se o espectro de Fourier do sinal ondulatório gerado pelas coordenadas do ponto a cada imagem.

Foram realizados 7 experimentos em P-GMAW padrão, e 5 experimentos em P-GMAW com corrente de destacamento. Será apresentado um experimento de cada.

\section{Resultados e Discussão}

\subsection{ExperimentoemP-GMAWcomCorrentedeDestacamento}

Para este experimento foi configurada uma curva característica no modo pulsado com os parâmetros da Tabela 1, referentes à forma de onda mostrada na Figura 7.

Tabela 1. Parâmetros configurados para o primeiro experimento.

\begin{tabular}{c|c}
\hline Parâmetros de Pulsação & Valores \\
\hline Corrente de base $\left(I_{b}\right)$ & $80 \mathrm{~A}$ \\
\hline Aumento de corrente $(\mathrm{Tau})$ & $0,20 \mathrm{~ms}$ \\
\hline Corrente de pulsação $\left(I_{p}\right)$ & $380 \mathrm{~A}$ \\
\hline Tempo de corrente de pulsação $\left(\mathrm{t}_{p}\right)$ & $3 \mathrm{~ms}$ \\
\hline Queda de corrente (Tau) & $0,2 \mathrm{~ms}$ \\
\hline Corrente de separação de gotas $\left(I_{g}\right)$ & $100 \mathrm{~A}$ \\
\hline Tempo de corrente de separação de gotas $\left(t_{g}\right)$ & $2 \mathrm{~ms}$ \\
\hline Frequência de base (fb) & $150 \mathrm{~Hz}$ \\
\hline Velocidade do arame & $5 \mathrm{~m} / \mathrm{min}$ \\
\hline Velocidade de soldagem & $9,5 \mathrm{~mm} / \mathrm{s}$ \\
\hline Vazão do gás de proteção & $15 \mathrm{~L} / \mathrm{min}$ \\
\hline Distância entre bico de contato e a peça de trabalho & $14 \mathrm{~mm}$ \\
\hline
\end{tabular}

O importante para esse experimento era encontrar parâmetros que permitissem uma soldagem pulsada sem que ocorressem curtos-circuitos. Outra questão relevante para a escolha dos parâmetros era a obtenção de um arco com pressão forte, ou seja, com um $\varphi$ inferior a 0,2 para que a oscilação da poça fosse mais influenciada pela oscilação do arco, segundo a Equação 1. Para aplicar essa equação, considera-se que $\mathrm{I}_{\mathrm{b}}$ é a corrente média 


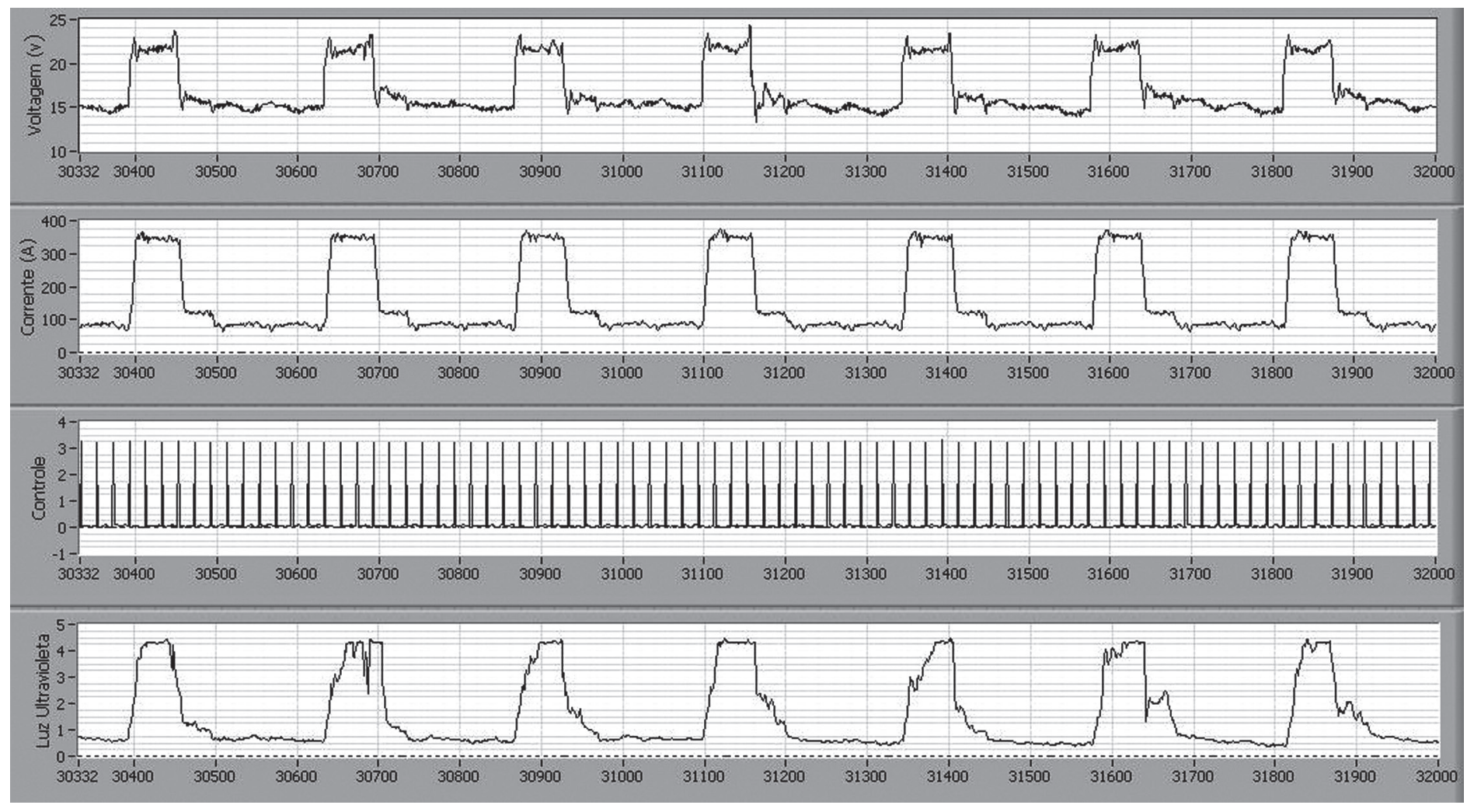

Figura 9. Amostra de sinais adquiridos. Os gráficos mostram na sequência de cima para baixo respectivamente os sinais de tensão e de corrente de soldagem, de pulsos de controle da câmera e de intensidade de luz ultravioleta emitida.

entre a corrente de base e a corrente de separação de gotas $I_{g}$, já que essa última está mais próxima da corrente de base que da corrente de pico. Nesse caso $I_{b}$ vale:

$$
\begin{gathered}
I_{b}^{\prime}=\frac{\left(80 \cdot 1 / f_{b}+100 \cdot t_{g}\right)}{1 / f_{b}+t_{g}} \\
I_{b}^{\prime}=\frac{\left(80 \cdot 1 / 150+100 \cdot 2 \cdot 10^{-3}\right)}{1 / 150+2 \cdot 10^{-3}}=84,61 \mathrm{~A}
\end{gathered}
$$

A frequência (f) de pulsação da corrente é de:

$$
f=\frac{1}{t_{p}+t_{g}+t_{b}}=\frac{1}{3 \cdot 10^{-3}+2 \cdot 10^{-3}+1 / 150}=85,71 \mathrm{~Hz}
$$

O valor de $\varphi$ é calculado como:

$$
\begin{gathered}
\phi=\left(I_{b}^{\prime} / I_{p}\right) \cdot f \cdot t_{b}^{\prime} \\
\phi=\left(\frac{84,61}{380}\right) \cdot 85,71 \cdot \frac{1}{150}+2 \cdot 10^{-3} \cong 0,165
\end{gathered}
$$

Observa-se pela figura 9, que não houve ocorrência de curto-circuitos no trecho de soldagem avaliado. Além disso, os cordões de solda produzidos mostraram morfologia satisfatória.

A Figura 9 mostra os sinais capturados de tensão, corrente, strobe ( sinal de controle) da câmera e intermitência luminosa. $\mathrm{O}$ sinal de intermitência foi utilizado para auxiliar na confirmação do sincronismo das imagens com os outros sinais.

Não foi construído um filtro eletrônico para a filtragem dos sinais para não gerar atrasos na captura dos mesmos. No entanto após a obtenção dos sinais, eles foram filtrados digitalmente para fins de processamento das informações.

A Figura 10 mostra o espectro de Fourier calculado para o sinal da tensão de soldagem adquirido durante um tempo correspondente às 500 primeiras fotos obtidas (o espectro da corrente foi bem semelhante e não será mostrado).

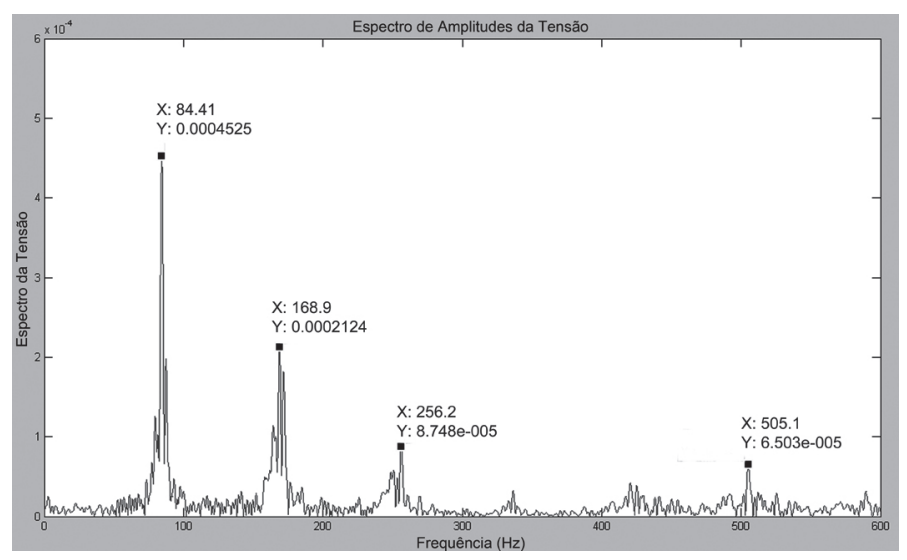

Figura 10. Espectro de frequências da tensão de soldagem. 


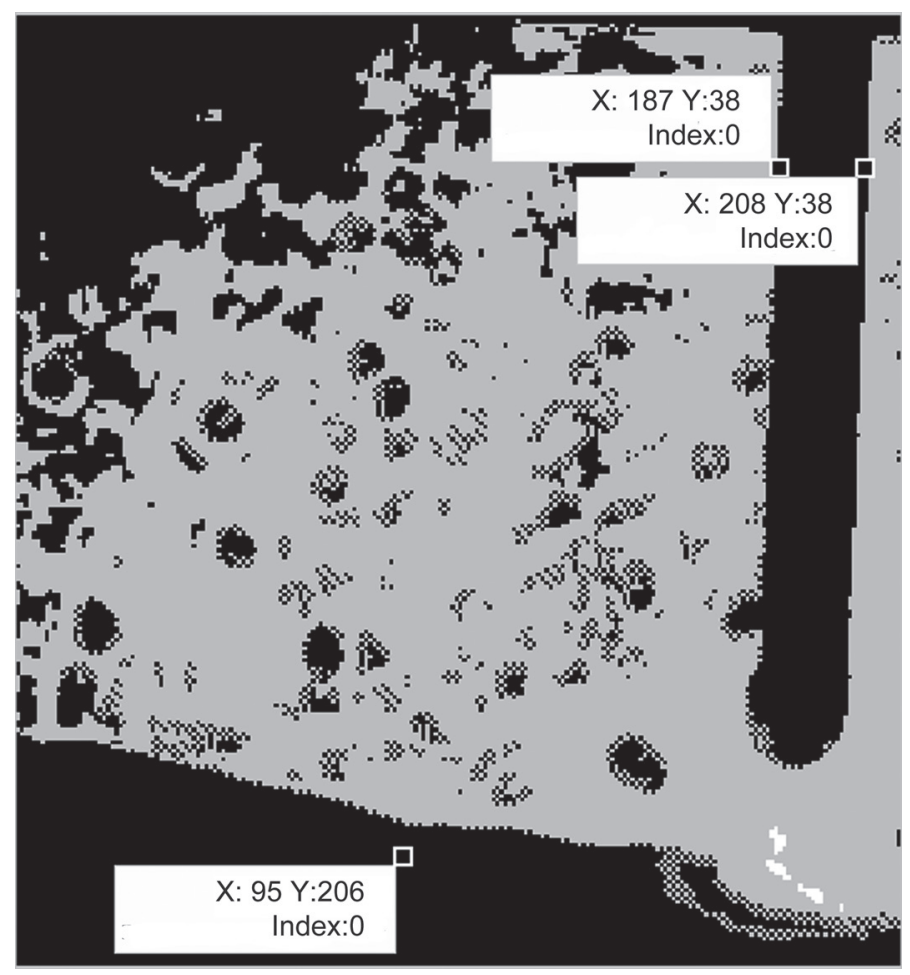

Figura 11. Primeira imagem para análise.

O espectro de frequência da tensão de soldagem mostra que a frequência mais significativa é de $84,4 \mathrm{~Hz}$, próximo ao selecionado na fonte de $85,7 \mathrm{~Hz}$. As demais frequências se tratam de harmônicas por serem múltiplos da frequência principal.

\subsection{Análise de imagens da poça de solda}

Analisaram-se os 500 primeiros quadros da poça de solda. A primeira imagem após tratamento em MATLAB pode ser vista na Figura 11.

$\mathrm{Na}$ imagem da Figura 11, pode-se conhecer o tamanho de cada pixel tendo como referência o diâmetro do arame que é de 1,2 mm. Com o diâmetro do arame de 22 pixels, o tamanho de cada pixel neste experimento é de 1,2/22 $=0,0545 \mathrm{~mm}$ aproximadamente. A imagem original tem 216x232 pixels e foi adquirida em 1000 fps. Primeiramente, foi analisada a oscilação de uma mesma parcela de massa. Por exemplo, iniciando no ponto de coordenada $X=95$. Após escolhido esse ponto na imagem, o programa em MATLAB procura a margem superior e armazena a coordenada deste pixel. Para a próxima imagem o programa procura novamente a margem superior e armazena a nova coordenada do pixel e assim sucessivamente. A união desses pontos forma um gráfico oscilatório, que corresponde à oscilação da crista da poça de solda na região que se deseja observar. Em 500 imagens, o deslocamento do ponto iniciando em $\mathrm{X}=95$ e considerando a velocidade linear da mesa de 9,5 $\mathrm{mm} / \mathrm{s}$ é de:

$$
d X=499 \cdot 10^{-3} \mathrm{~s} \cdot 9,5 \frac{\mathrm{mm}}{\mathrm{s}}=4,7405 \mathrm{~mm}
$$

Esse deslocamento corresponde na imagem a um deslocamento em termos de pixels de:

$$
d P=\frac{d X}{1,2 / 22} \cong 87
$$

A oscilação do ponto com coordenada inicial $X=95$ pode ser vista na Figura 12.

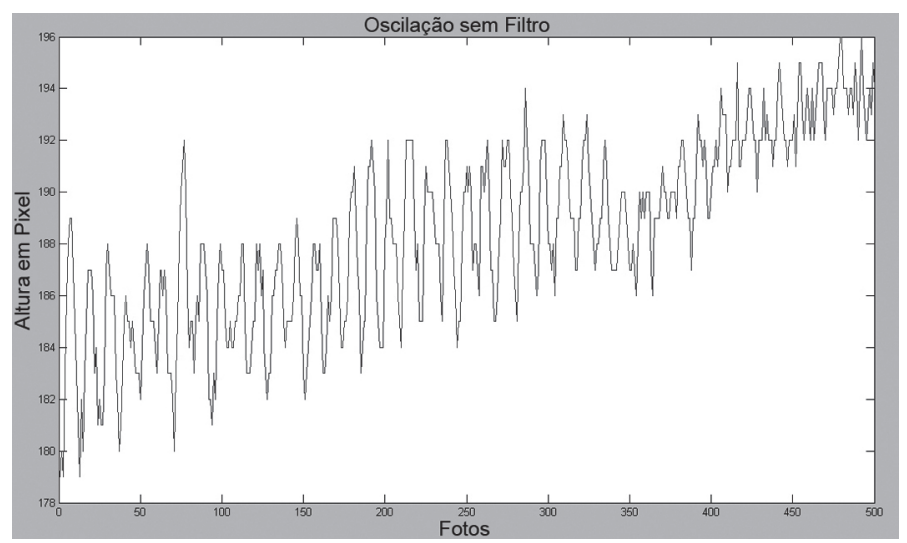

Figura 12. Oscilação do ponto localizado inicialmente na coordenada $X=95$.

Considerando que a origem da coordenada Y em uma imagem capturada é o pixel mais à esquerda, localizada na parte superior da imagem, na primeira linha, observa-se que o resultado da busca pela interface poça-gás de proteção apresenta valores menores de coordenada $\mathrm{Y}$ para amplitude positiva e valores maiores, para amplitude negativa. Para ter como resultado um efeito mais natural, o gráfico da Figura 12 tem os valores invertidos, ou seja, se o nível mais alto de oscilação tinha o valor em MatLab de $\mathrm{Y}=179$ pixels, e o mais baixo de $\mathrm{Y}=196$ pixels, para gerar o gráfico esses valores e os demais foram invertidos de modo a se ter o efeito real da oscilação.

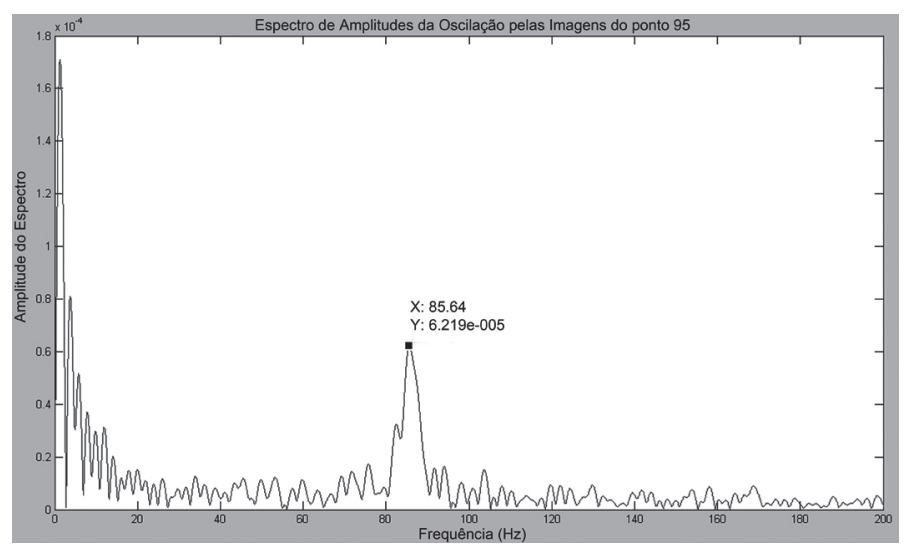

Figura 13. Frequência de oscilação do ponto 95 igual a 85,64 $\mathrm{Hz}$.

Observa-se que a amplitude de oscilação diminui com o deslocamento do ponto na direção $\mathrm{X}$ da imagem, também 
aumentando sua frequência. Por outro lado, para verificar frequências de oscilação de regiões fixas do cordão foram analisados três pontos com coordenadas $\mathrm{X}$ fixas e distintas, sendo um mais próximo do arco, um intermediário no cordão e outro mais distante. Para a análise desses pontos foi utilizado o filtro de Sobel, uma vez que este apresentou melhores resultados em regiões mais próximas do arco. Para representar a região localizada a uma distância intermediária do arco, escolheu-se a coordenada $X=95$. A Figura 13 mostra o espectro de Fourier calculado para a variação da coordenada $\mathrm{Y}$ da crista da onda referente à coluna de pixels de coordenada $X=95$.

Para representar uma região mais próxima do arco, escolheu-se a coluna de pixels com coordenada $\mathrm{X}=138$, para cujos resultados de variação da coordenada $Y$, na interface poça-gás de proteção, se calculou o espectro de Fourier mostrado na Figura 14.

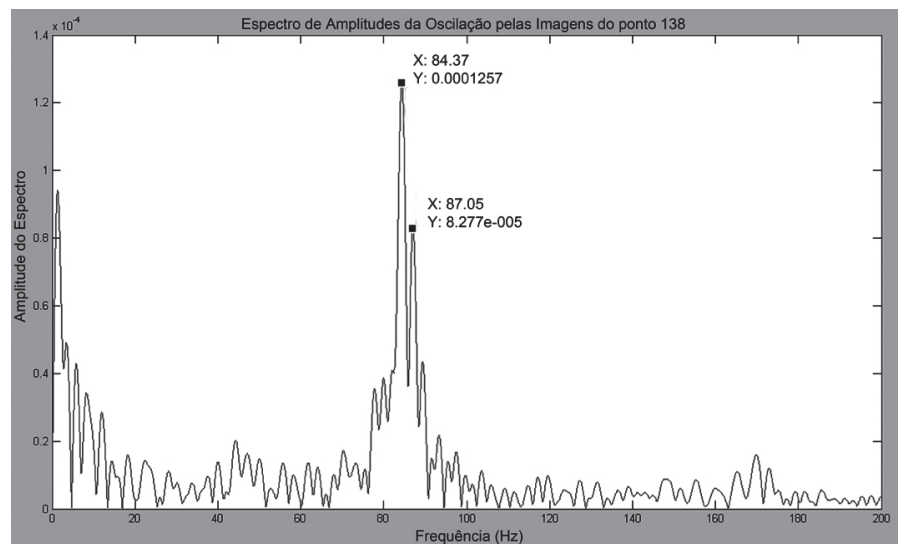

Figura 14. Frequência de oscilação em $X=138$ com picos em $84,37 \mathrm{~Hz}$ e $87,05 \mathrm{~Hz}$.

Já para representar a região da poça mais distante do arco, escolheu-se a coluna de pixels com coordenada $\mathrm{X}=08$, sobre a qual se aplicou o algoritmo de busca da coordenada $\mathrm{Y}$ correspondente à crista da onda. Os resultados para as 500 primeiras fotos possibilitaram o cálculo do espectro de Fourier mostrado na Figura 15.

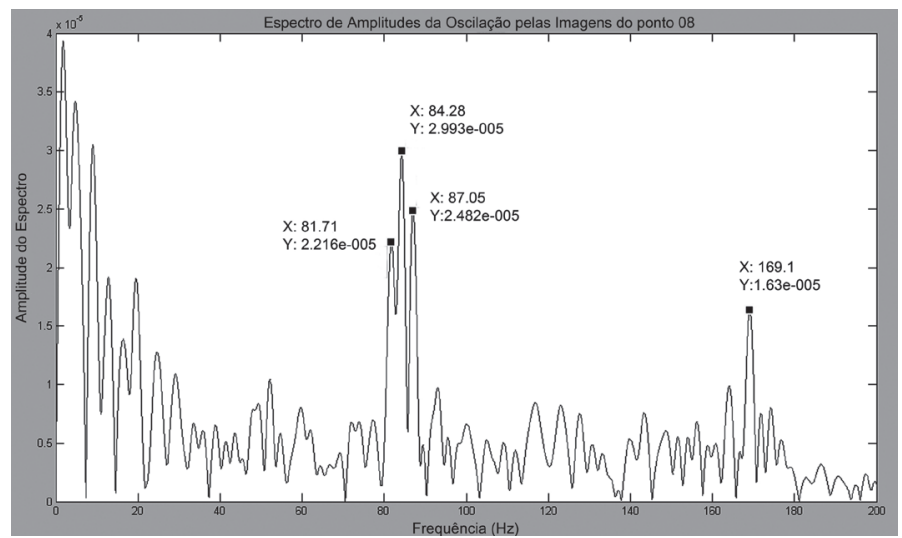

Figura 15. Frequência de oscilação em $X=08$ entre $81,71 \mathrm{~Hz}$ e $87,05 \mathrm{~Hz}$ e um destaque em $169,1 \mathrm{~Hz}$.

Como o ponto 138 está mais próximo do arco, é de se esperar que a frequência dele se aproxime mais à frequência do arco, o que foi observado. Nos pontos mais distantes a temperatura é um pouco menor, o que faz com que a tensão superficial aumente e é de se esperar que a frequência de oscilação aumente também.

\subsection{Experimento em P-GMAW convencional (sem corrente de destacamento)}

Os parâmetros de corrente configurados para este experimento são apresentados na Tabela 2.

Tabela 2. Parâmetros configurados para o segundo experimento.

\begin{tabular}{c|c}
\hline Parâmetros de Pulsação & Valores \\
\hline Corrente de base $\left(\mathrm{I}_{\mathrm{b}}\right)$ & $80 \mathrm{~A}$ \\
\hline Aumento de corrente $(\mathrm{Tau})$ & $0,20 \mathrm{~ms}$ \\
\hline Corrente de pulsação $\left(\mathrm{I}_{\mathrm{p}}\right)$ & $380 \mathrm{~A}$ \\
\hline Tempo de corrente de pulsação $\left(\mathrm{t}_{\mathrm{p}}\right)$ & $3,2 \mathrm{~ms}$ \\
\hline Queda de corrente $(\mathrm{Tau})$ & $0,2 \mathrm{~ms}$ \\
\hline Corrente de separação de gotas $\left(\mathrm{I}_{\mathrm{g}}\right)$ & $80 \mathrm{~A}$ \\
\hline Tempo de corrente de separação de gotas $\left(\mathrm{t}_{\mathrm{g}}\right)$ & $1 \mathrm{~ms}$ \\
\hline Frequência de base $\left(\mathrm{f}_{\mathrm{b}}\right)$ & $75 \mathrm{~Hz}$ \\
\hline Velocidade do arame & $4 \mathrm{~m} / \mathrm{min}$ \\
\hline Velocidade de soldagem & $8,0 \mathrm{~mm} / \mathrm{s}$ \\
\hline Vazão do gás de proteção & $15 \mathrm{~L} / \mathrm{min}$ \\
\hline Distância entre bico de contato e a peça de & $14 \mathrm{~mm}$ \\
trabalho & \\
\hline
\end{tabular}

Com esses parâmetros a frequência do pulso de corrente é de aproximadamente $57 \mathrm{~Hz}$ :

$$
\begin{aligned}
& f=\frac{1}{t_{p}+t_{g}+t_{b}}=\frac{1}{3.2 \cdot 10^{-3}+1 \cdot 10^{-3}+1 / 75} \cong 57 \mathrm{~Hz} . \\
& \text { O fator é de aproximadamente } 0,172:
\end{aligned}
$$

$$
\phi=\left(\frac{I_{b}}{I_{p}}\right) \cdot f \cdot t_{b}=\left(\frac{80}{380}\right) \cdot 57 \cdot\left(\frac{1}{75}\right)+1 \cdot 10^{-3}=0,172
$$

Utilizando a metodologia desenvolvida, investigou-se o comportamento oscilatório para as primeiras 301 imagens. A velocidade de alimentação do arame foi de $4 \mathrm{~m} / \mathrm{min}$ e da mesa foi de $8 \mathrm{~mm} / \mathrm{s}$. A frequência fundamental de oscilação do sinal de tensão foi de $56,57 \mathrm{~Hz}$ e da corrente foi de $56,51 \mathrm{~Hz}$.

O espectro de frequência de oscilação das imagens também foi obtido. Primeiro examinou-se a oscilação de uma parcela de massa no cordão e depois a oscilação de regiões fixas. Os pontos escolhidos podem ser vistos na Figura 16. Os resultados mostraram que aparecem frequências mais significativas durante todo o cordão na faixa de 56 a $58 \mathrm{~Hz}$ e de 113 a $115 \mathrm{~Hz}$. 


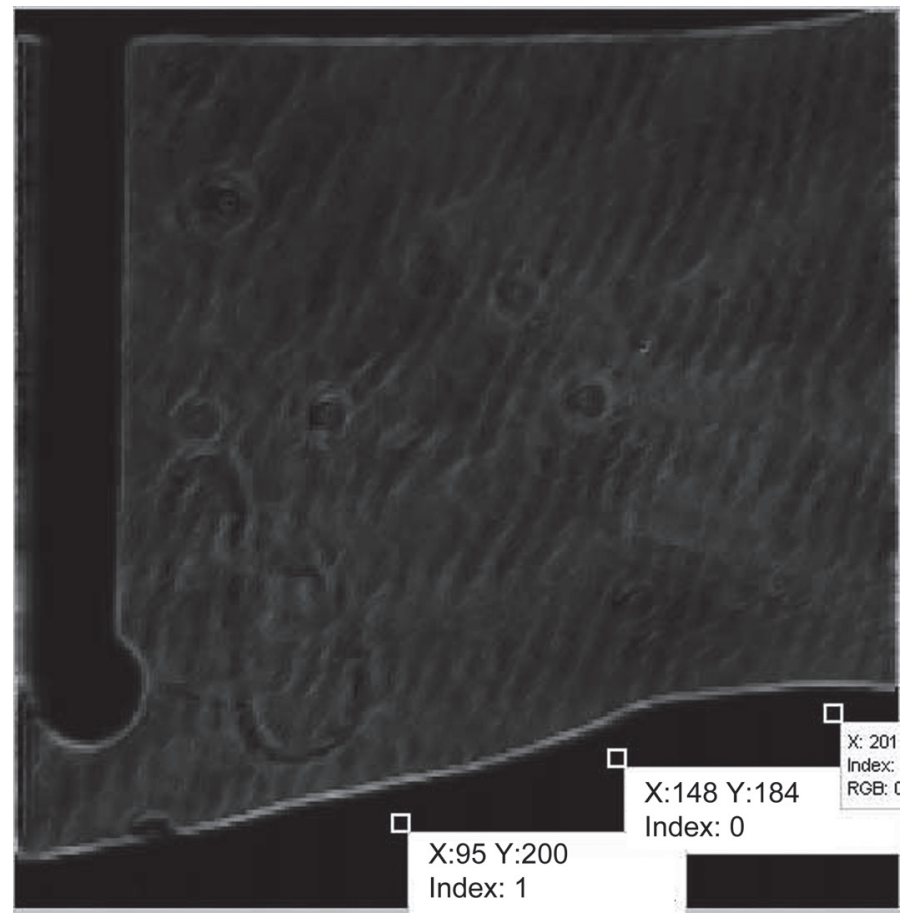

Figura 16. Pontos escolhidos em foto processada com filtro de Sobel.

Foi observado que a frequência fundamental se mantém próxima de $57 \mathrm{~Hz}$ e com amplitude maior e depois aumenta a frequência para $114 \mathrm{~Hz}$ e diminui a amplitude. A frequência de 114 por ser o dobro de 57 pode ser considerada uma segunda harmônica, que aparece na oscilação da poça.

\section{Conclusões}

Foi desenvolvida uma metodologia computacional para obtenção da oscilação da poça por análise dos sinais envolvidos no processo, bem como pelas imagens fotografadas. E esse método permitiu calcular as frequências de oscilação da poça de solda utilizando apenas $1000 \mathrm{fps}$.

A iluminação por perfilografia permitiu analisar a oscilação da poça através da crista das ondas formadas. Conseguiu-se eliminar bastante a luminosidade do arco utilizando um tempo de exposição de $10 \mu \mathrm{s}$ e um filtro óptico na região de $633 \mathrm{~nm}$. Mesmo assim durante os picos de tensão o arco atrapalha a observação de pontos mais próximos do arame. Por isso procurou-se analisar pontos que não fossem ofuscados pelo arco.

Os resultados mostraram que o arco realmente exerce pressão na poça, conforme estabelecido na literatura, e essa passa a oscilar, no estado estacionário, com as mesmas frequências excitadas pelo arco, que no caso em questão, tem forma semelhante a uma onda quadrada. Observou-se que a amplitude de oscilação da poça na região próxima à coluna do arco foi maior que em pontos mais distantes. Inversamente, as frequências medidas foram maiores nos pontos mais distantes do que nos pontos mais próximos

Como a fonte de calor está se movendo, ao se afastar da região excitada tanto pelo arco como pelo metal transferido, há uma redução na massa líquida disponível à oscilação, devido à solidificação gradual do metal fundido. Considerando que a vibração depende do balanço de forças de tensão superficial e gravitacional atuando no metal líquido, em conjunto com as forças de inércia, a redução da massa de metal líquido implica em redução das forças gravitacionais e inerciais, enquanto que o resfriamento resulta provavelmente em um aumento das forças de tensão superficial, o que implica em uma rigidez maior no sistema dinâmico da poça e a faz oscilar com frequência maior e com amplitude menor. Um estudo mais detalhado sobre o fenômeno pode ser visto no trabalho de Ramos, E.G. [4].

A avaliação de incertezas para o método seria necessária para a quantificação do erro de medida resultante. Entretanto, a ausência do cálculo de incerteza não invalida a metodologia, uma vez que os resultados obtidos tanto em termos de cálculo de espectro de frequências com base em sinais de tensão, como com base em análise de imagens, apresentaram-se em conformidade com as frequências de pulsação ajustadas na fonte de soldagem, com erros bastante pequenos. $\mathrm{O}$ que se observou pelo perfil da poça de solda é que ela oscilou principalmente com a frequência fundamental de excitação imposta pela fonte de soldagem, o que já era esperado considerando que a poça líquida comporta-se como um sistema dinâmico excitado por um sinal periódico.

\section{Referências Bibliográficas}

[1] RIBEIRO, A.F.M. Automated off-line programming for rapid prototyping using gas metal arc welding. PhD Thesis, Cranfield University, UK, 1995.

[2] RIBEIRO, A.F.M., NORRISH, J. Practical case of rapid prototyping using gas metal arc welding. In: 5th International Conference on Computer Technology in Welding (TWI - The Welding Institute, Crampton Printers), 15-16 June 1994, Paris, França, Paper 55.

[3] RIBEIRO, A.F.M., NORRISH, J. Practical case of rapid prototyping using gas metal arc welding. In: 6th International Conference on Computer Technology in Welding (TWI - The Welding Institute, Abington Publishing), 9-12 June 1996, Lanaken, Belgium. Paper 60.

[4] RAMOS, E. G. (2011). Análise da Oscilação da Poça de Solda em GMAW por Meio de Processamento de Imagens Obtidas por Perfilografia. Dissertação de Mestrado em Sistemas Mecatrônicos, Publicação ENM 02/2011, Departamento de Engenharia Mecânica, Universidade de Brasília, Brasília, DF, 98p.

[5] Yudodibroto, R. B. Y. B. Liquid Metal Oscillation and Arc Behaviour during Welding. Tese (Doutorado) - Delft University of Technology.

[6] Ghosh, P. K., Gupta, S. R. e Randhawa, H. S. Characteristics and criticality of bead on plate deposition in pulsed current vertical-up GMAW of steel. Int. J. Join. Mater., 1999.

[7] Randhawa, H. S, Ghosh, P. K., Gupta, S. R., Some basic aspects of geometrical characteristics of pulsed current vertical- 
up GMA weld, ISIJ Int. 2000.

[8] Ghosh, P. K., et. al. Thermal and metal transfer behaviours in pulsed current GMA weld deposition of Al-Mg alloy. STWJ. 2006.

[9] Ghosh, P. K., et. al. Arc characteristics and behaviour of metal transfer in pulsed current GMA welding of aluminium alloy. J. Mater. Process. Technol. 2007.

[10] Ghosh, P.K., et. al. Arc characteristics and behaviour of metal transfer in pulsed current GMA welding of stainless steel. Journal of Materials Processing Technology 209, 2008.

[11] Lancaster, J. F., Metallurgy of Welding, 1987.

[12] Rhee, S. e Asibu, E. K. Analysis of arc pressure effect on metal transfer in gas metal arc welding, J. Appl. Phys, 1991.

[13] Xiao, Y. H. e Den Ouden, G., Direct observation of GTA weld pool oscillation, Proceeding of the 3rd International Conference on Trends in Welding Research, Gatlinburg, 1992.

[14] Yoo, C. D. e Richardson, R. W., An experimental study on sensitivity and signal characteristics of weld pool oscillation, Transactions of the Japan Welding Society, 1993.

[15] Bicknell, A., Smith, J. S. e Lucas, J., Arc voltage sensor for monitoring of penetration in TIG welds, IEE Proc. Sci. Meas. Technol., Vol. 141, No. 6, 1994.

[16] Bálsamo P. S. S., et. al., Development of an experimental technique for studying metal transfer in welding: synchronized shadowgraphy, Int. J. for the Joining of Materials, 2000.

[17] Weichel, H., Laser Beam Propagation in the Atmosphere, SPIE, 1990.

[18] Steen, W. e Watkins, K., Laser Material Processing, Springer, 2003.

[19] Hecht, E., Optics, Ed. Addison Wesley, San Francisco, USA, 2002.

[20] Lopera, J. E. P., et. al., Uso da técnica de perfilografia para visualização dos modos de transferência metálica no processo de soldagem GMAW usando uma câmera CMOS de alta velocidade, COBEF 2011, Universidade de Brasília.

[21] Fronius, Manual de operação da fonte de soldagem, RCU 5000 i, 2008.

[22] Gonzalez, R. C.; Woods, R. E. Processamento de Imagens Digitais. Rua Pedroso Alvarenga, 1245 - São Paulo, SP - Brasil: Editora Edgard Blücher, 2007. 